\title{
NMR Measurements for Solid Polarized Targets at Jefferson Lab
}

\author{
James D. Maxwell ${ }^{*}$ \\ Thomas Jefferson National Accelerator Facility, Newport News, VA USA \\ E-mail: jmaxwellajlab.org
}

\begin{abstract}
Jefferson Lab's solid polarized targets rely on Nuclear Magnetic Resonance techniques to measure the enhanced polarization provided under Dynamic Nuclear Polarization. Upcoming polarized target experiments in JLab's Hall B present challenging conditions which would benefit from improvements to traditional NMR techniques. For decades, JLab has utilized Liverpool Q Meters for NMR measurements, but these are aging and no longer produced. The polarized target group at Bochum has successfully produced replacement Q Meters with modern components, and we are following their example, exploring new designs for both an analog and a digital Q Meter system. Unlike recent experiments in Halls A and C, the new Hall B target will require external NMR coils, resulting in a weaker signal. In addition, two separate target cells will be utilized, each held at different magnetic fields, allowing them to be polarized in opposing directions, but complicating the NMR measurement. We present the challenges presented by the new Hall B target, lay out plans for improvements to our NMR system, and show results of initial tests of our designs.
\end{abstract}

23rd International Spin Physics Symposium - SPIN2018 -

10-14 September, 2018

Ferrara, Italy

\footnotetext{
* Speaker.

${ }^{\dagger}$ On behalf of the Jefferson Lab Target Group and CLAS12 Polarized Target Collaboration
} 


\section{Introduction}

Dynamic nuclear polarized (DNP) targets are crucial tools for Jefferson Lab's polarized scattering experiments [1], capable of providing in excess of $95 \%$ polarization of protons (in $\mathrm{NH}_{3}$ ) or $50 \%$ polarization of deuterons (in $\mathrm{ND}_{3}$ ). DNP takes advantage of the very high electron polarization $(P=\tanh (\mu B / k T)))$ at a practical temperature $(T \sim 1.4 \mathrm{~K})$ and magnetic field $(B \sim 5 T)$, to polarize nearby nuclei via flip-flop transitions in the electron-spin-nucleus-spin system. These transitions are driven by microwave radiation of frequency close to the electron spin resonance frequency less or more the nuclear Larmor frequency ( $\left.\omega_{\mathrm{esr}} \pm \omega_{\mathrm{nmr}}\right)$.

Nuclear Magnetic Resonance (NMR) methods are used to measure the polarization achieved in a target sample. With a coil of inductance $L_{0}$ embedded in the sample perpendicular to the polarizing field $B$, an induced field $B_{0}$ at the Larmor frequency $\omega_{0}$ drives spin flips, causing transitions of the Zeeman-splitting energy levels, $\hbar \omega_{0}=g \mu B$. Depending on the average orientation of the spins to the field, these spins flips absorb or emit energy as they interact with the field generated by the inductor and its electrical circuit. The spin system's response to the induced RF field $B_{0}$ is its magnetic susceptibility, $\chi(\omega)$, and is a function of the RF frequency $\omega$. The magnetic susceptibility consists of a dispersive term $\chi^{\prime}(\omega)$ and an imaginary absorptive term $\chi^{\prime \prime}(\omega)$. This absorptive term can be integrated in frequency to give a proportional measure of the target polarization $P$ :

$$
\chi(\omega)=\chi^{\prime}(\omega)-i \chi^{\prime \prime}(\omega), \quad P=K \int_{0}^{\infty} \chi^{\prime \prime}(\omega) d \omega
$$

Here $K$ contains information on the target nucleus as well as the properties of the NMR system. Since $\chi^{\prime \prime}(\omega)$ is non-zero only in a small range around the Larmor frequency, the integral only need be performed very close to center. The inductance of the embedded coil couples to the magnetic susceptibility, via a filling factor $\eta$,

$$
L_{C}(\omega)=L_{0}[1+4 \pi \eta \chi(\omega)]
$$

so that a measurement that isolates the real part of the complex impedance of the coil versus frequency will give a proportional measure of the polarization.

Polarized target applications, such as the scattering experiments at Jefferson Lab, have typically used Liverpool Q-meters [2] to perform NMR measurements. Traditionally, the Q-meter observes a tank (LCR) circuit with the resistor and capacitor within the Q-meter connected via a $\lambda / 2$ cable to the inductor embedded in the material at $1 \mathrm{~K}$, as shown in figure $1 \mathrm{a}$. The tank circuit's adjustable capacitor allows the tuning of the circuit so that the resonant frequency, $\omega_{0}=1 / \sqrt{L C}$, can be set to the Larmor frequency of the nucleus of interest. The Q-meter's key component is a balanced ring modulator, a type of frequency mixer which compares the signal from the tank circuit to the reference excitation RF frequency and returns the real portion of the signal—when the signal and reference match phase.

In the course of a measurement, the reference signal is swept through frequency, starting below the Larmor frequency and increased through it in discrete steps. For a well-tuned circuit, the Qmeter response results in a symmetric background curve versus frequency, known as the Q-curve, from reactive complex impedance below and above the resonant frequency. Any effect from the material polarization, such as the proton thermal equilibrium polarization signal seen in figure 2 , 


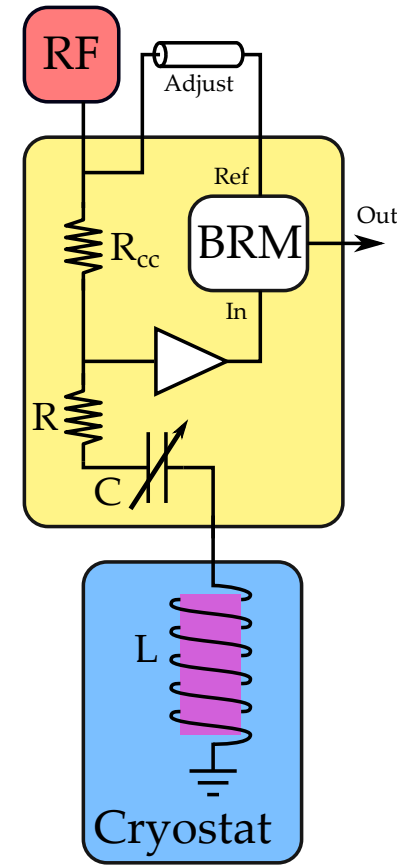

(a) Traditional Q-meter NMR

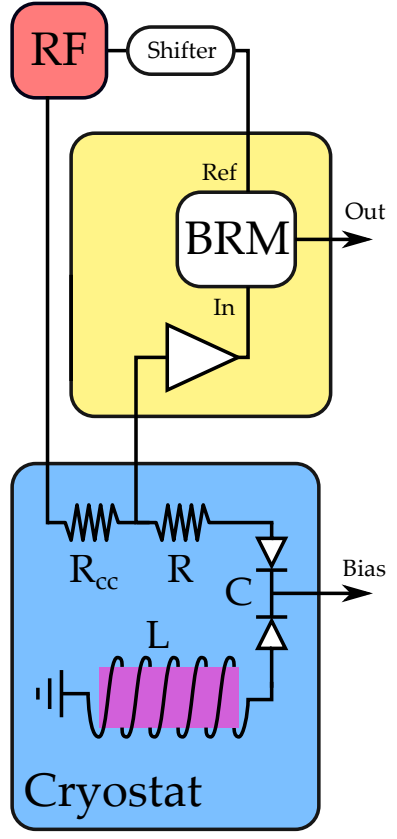

(b) "Cold" Q-meter NMR

Figure 1: Diagrams showing the traditional NMR layout, and the new, "cold" circuit NMR layout, which includes varactor-diode tuning capacitors and an electronic phase shifter.

is integrated by first removing this background Q-curve (or baseline). Thermal equilibrium (TE) measurements such as this one at known temperature and magnetic field allow the calibration of the signal area when the polarization is enhanced.

The tuning of the Q-meter NMR circuit in the Liverpool system involves three basic steps. First, the length of the cable between the coil and the resistor and adjustable capacitor must be chosen to be an integer multiple of half the resonant wavelength $(n \lambda / 2)$, removing the effect of the cable's impedance. Then, the tank circuit is tuned to $\omega_{0}$ by adjusting the capacitance in the Q-meter. Finally, the phase between the tank signal and the reference signal must be matched by adjusting the length of cable that the reference takes into the mixer, centering the real portion of the signal at $\omega_{0}$.

\subsection{NMR for the CLAS12 Polarized Target}

A new polarized target is being developed for the new CLAS12 detector package, building on the success of the polarized target program in Hall B during Jefferson Lab's $6 \mathrm{GeV}$ era [3]. The new polarized target system will fit inside the 5 T CLAS12 solenoid, and will accommodate two target sample cells in the beam simultaneously. These cells, $2 \mathrm{~cm}$ long by $2 \mathrm{~cm}$ in diameter, will sit on the axis of the beam, in the center of the target cryostat, separated horizontally by roughly $3 \mathrm{~cm}$. In a scheme first proposed by S. Kuhn of ODU, we will use 2 sets of shim coils to increase and decrease the magnetic field by up to 80 Gauss over the extent of either cell, allowing a single microwave frequency to be used to polarize each cell to opposite polarities, as shown in figure 3 . 


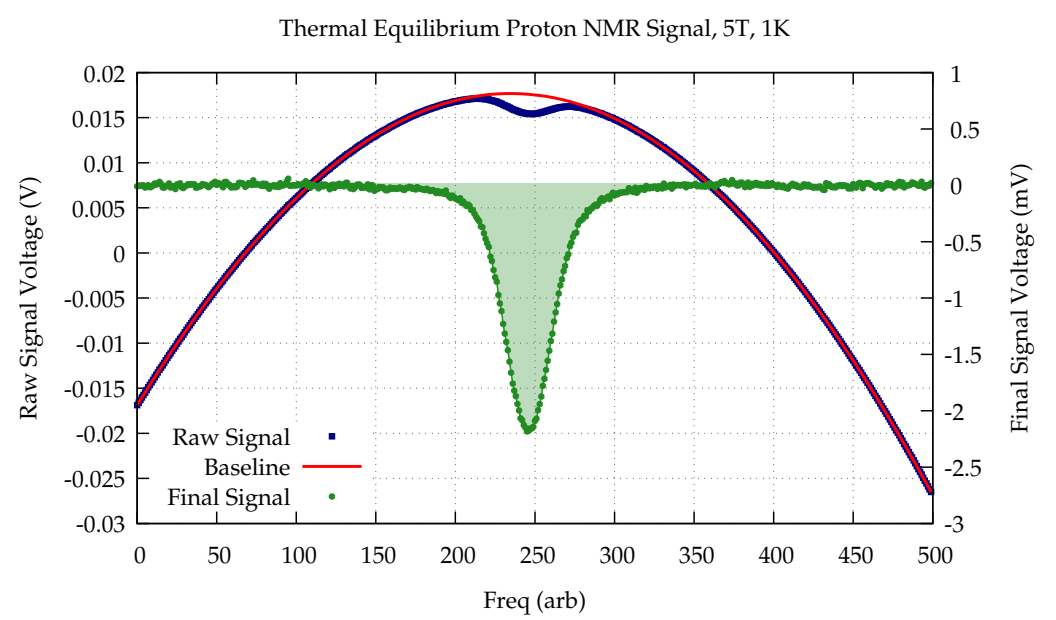

Figure 2: A Proton TE signal at $5 \mathrm{~T}$ and roughly $1 \mathrm{~K}$, showing the raw Q-meter response in blue, a polynomial fit to the baseline in red, and the resulting NMR signal after subtraction in green.

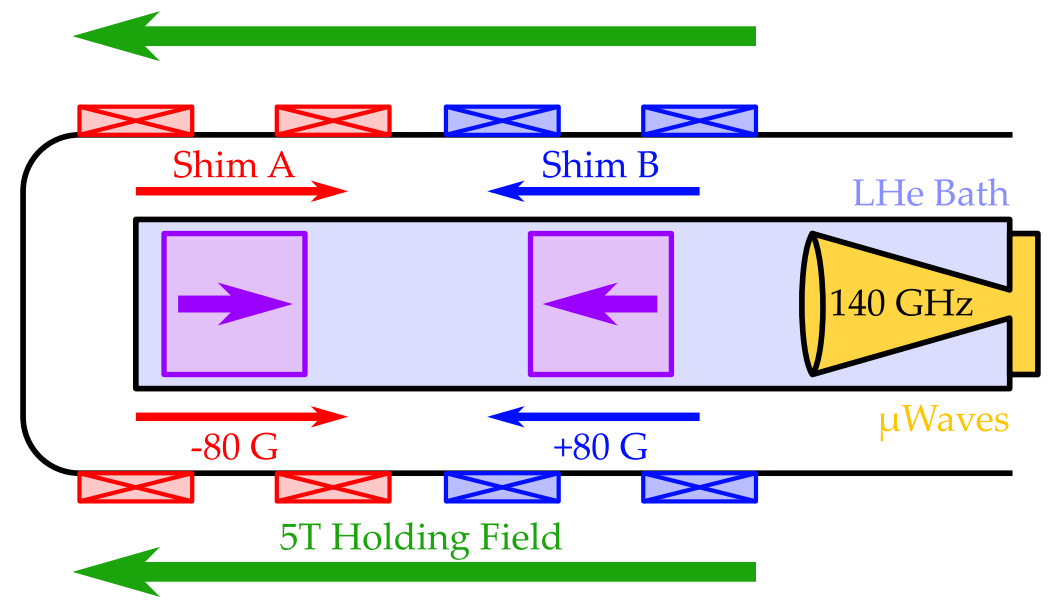

Figure 3: Double cell, opposing polarization configuration, with two sets of shim coils increasing and decreasing the magnetc field in the region of either cell to allow polarization using a single microwave frequency. This diagram shows the NMR test layout; during experiments in Hall B, the microwaves will be introduced from above to accommodate the passage of the electron beam.

A two-cell, opposed-polarization configuration has been successfully implemented at SMC [4] and more recently COMPASS [5], however, they both utilized a single magnetic field and two different microwave frequencies. The use of a single microwave frequency requires precise control over the shimmed fields and the frequency of the NMR measurement to operate smoothly. In the case of a traditional static polarizing field, the microwave frequency is chosen to maximize the polarization, and the optimal frequency changes as the material accumulates radiation damage [6, 7]. With a static microwave frequency, the field of each cell will need to be changed independently as dose is accumulated. As the field changes, so does the NMR frequency. The NMR system must 
be able to be tuned remotely for such a scheme to work in an experimental setting, and this is not possible in the traditional system with rotary capacitors and cable length adjustment of the phase.

\section{Development}

We are pursing a number of improvements to the solid polarized target NMR system to be used in the CLAS12 polarized target. We are revisiting the "cold" circuit NMR technique which moves the resistor and tuning capacitor into the cryostat [8], as seen in figure $1 \mathrm{~b}$, removing the need for a $\lambda / 2$ cable between those components and the coil, and reducing thermal noise. To this non-resonant-cable style circuit, we have introduced varactor-diode variable capacitors to allowing remote tuning of the tank circuit [9]. To avoid the need to change the length of cable for phase matching we have included commercially available electronic phase-shifters.

We have produced cold NMR circuit boards for use in these tests, such as the one seen in Figure 4. These boards include two MACOM MA46 varactor diodes [10] which provide between roughly 0.6 and $8 \mathrm{pF}$ of capacitance when provided a voltage bias up to $14 \mathrm{~V}$. These GaAs varactors were tested below $4 \mathrm{~K}$ and at high magnetic field to ensure their successful operation for our application. A fixed capacitor is used in parallel with the tuning capacitors to bring the circuit's tune near $213 \mathrm{MHz}$. To accommodate the cold circuit, the Liverpool Q-meter needs to be altered, bypassing the internal portion of the tank circuit. In cold-circuit mode, the reference signal is split at the generator to be sent directly to the cold board and to the Q-meter's mixer via the phase shifter.

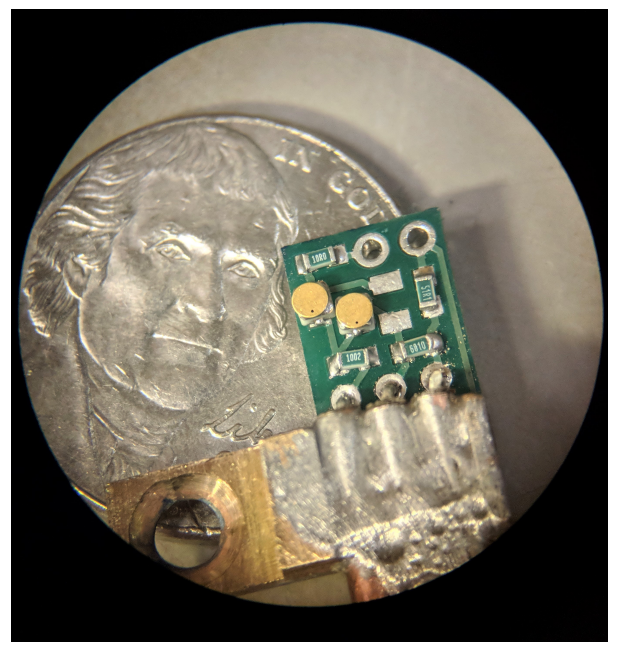

Figure 4: New cold NMR circuit, including GaAs varactor diodes, with American nickle for scale.

The demonstration and measurement of opposing polarization in two cells using a single microwave frequency is a key goal of our effort. The local magnetic field in the region of the two cells must be both uniform and precisely-controlled to permit polarization of the sample, so simulation of the shim coils was undertaken by collaborator V. Lagerquist of Old Dominion University. This simulation can suggest the best coil geometry and currents needed to produce the desired fields, within practical constraints. This is not only helpful in polarizing for NMR tests, but will be crucial 
in an experimental context, where the current in each of the 4 coils must be changed in relation to one another as optimal field for polarization changes with dose.

\subsection{Tests of Cold NMR at 5 T, $77 \mathrm{~K}$}

To allow an accelerated development cycle and reduce test costs, we have undertaken tests of NMR at $77 \mathrm{~K}$, rather than the $1.4 \mathrm{~K}$ at which DNP is typically performed. Using a $5 \mathrm{~T}$ warm-bore solenoid, we can perform NMR tests under a liquid nitrogen bath that extends into the center of the solenoid. At these temperatures, we can expect polarization signals to be over 50 times smaller than we would see at $1.4 \mathrm{~K}$, however the difficulty in observing small signals is somewhat offset by the ease of use of a simple, open bath a liquid nitrogen for cooling. To further facilitate tests focused on NMR performance, we use epoxy in place of traditional target material, which can have coils directly embedded within it to maximize coupling, and can be doped with TEMPO [11] to allow polarization via DNP. A discussion of our first demonstration of this $77 \mathrm{~K}$ approach to NMR tests is found in previous proceedings [12].

To observe the polarization in both cells simultaneously, NMR coils coupled to both cells were connected in parallel. With this configuration, a single NMR cold board could observe the signal contributions from both cells. Figure 5 shows example NMR signals using two epoxy sample cells at thermal equilibrium at $5 \mathrm{~T}$. These signals were taken using a set of NMR coils mounted on the exterior of the sample holders, much like the final Hall B target design. Figure 5a shows the combined TE signal from both samples when the shim coils are off. With the shim coils energized, the signals separate as their corresponding Larmor frequencies shift, as seen in Figure 5b. While these separated signals, each roughly half the amplitude of the combined signal, are clearly pushing the signal-to-noise limit of these measurements, even at $77 \mathrm{~K}$ they are distinct enough to see.

With distinct thermal equilibrium signals visible from each cell, we next attempted dynamic nuclear polarization of the two cells. To determine the necessary magnetic fields for each cell, we first dynamically polarized the samples both positively and negatively, measuring the microwave frequency needed for each. The driving microwave frequency was set to the average of these two frequencies, and the frequency difference between the average and the individual frequencies for positive and negative polarization was used to calculate the corresponding magnetic field shift required for either polarization. The shim simulation returned the currents needed in all 4 coils to produce these positive and negative polarizing magnetic fields.

Figure 6 shows a successful measurement of opposing polarizations in two cells via a single NMR coil. The two peaks seen in Figure 5b now point in opposite directions, indicating opposite polarizations. To operate the microwave tube at its optimal power setting, the center magnetic field was shifted to roughly $4.88 \mathrm{~T}$, corresponding to a proton Larmor frequency of $207.38 \mathrm{MHz}$. In this case, the positive and negative polarizing frequencies were found at $136.89 \mathrm{GHz}$ and $136.59 \mathrm{GHz}$ respectively, so the microwaves were set to $136.74 \mathrm{GHz}$ to polarize with the shims energized to increase and decrease the field by roughly 50 Gauss. Here the sweep modulation of the NMR was increased from $400 \mathrm{kHz}$ to $800 \mathrm{kHz}$ to better encompass both peaks. In this case the coils are embedded in the epoxy material to increase the signal size, rather than external. For these measurements, the thermal equilibrium signal was roughly $0.05 \mathrm{mV}$ in amplitude, so the enhancement from DNP is only about a factor of two. These clear and opposing peaks from adjacent material cells together in a single NMR signal represent a valuable proof-of-concept for this technique. 


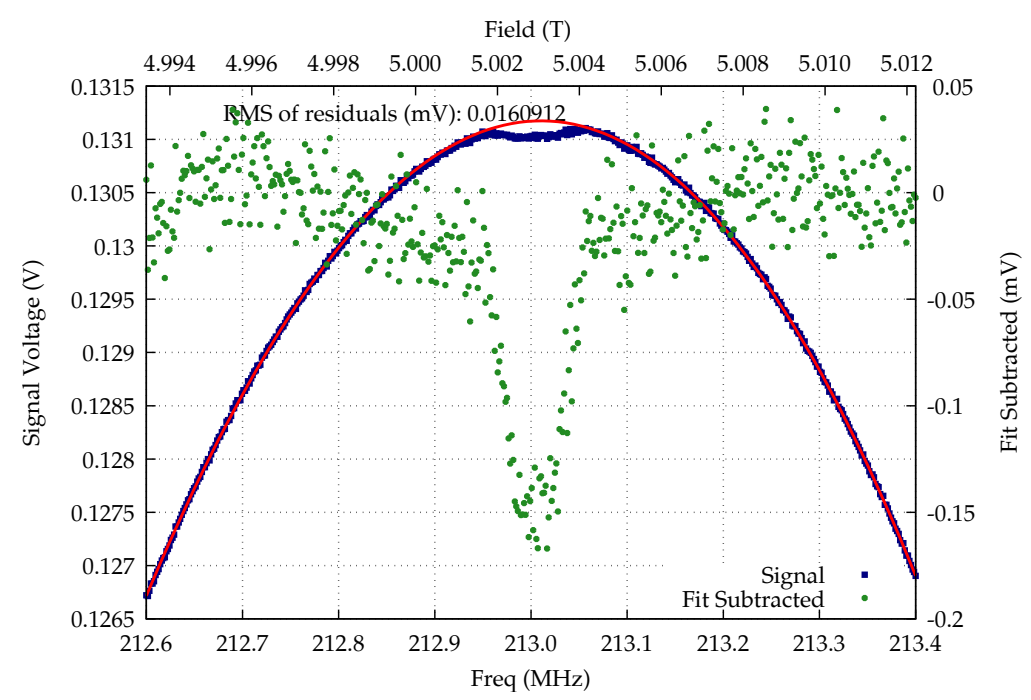

(a) Combined signal with shims off.

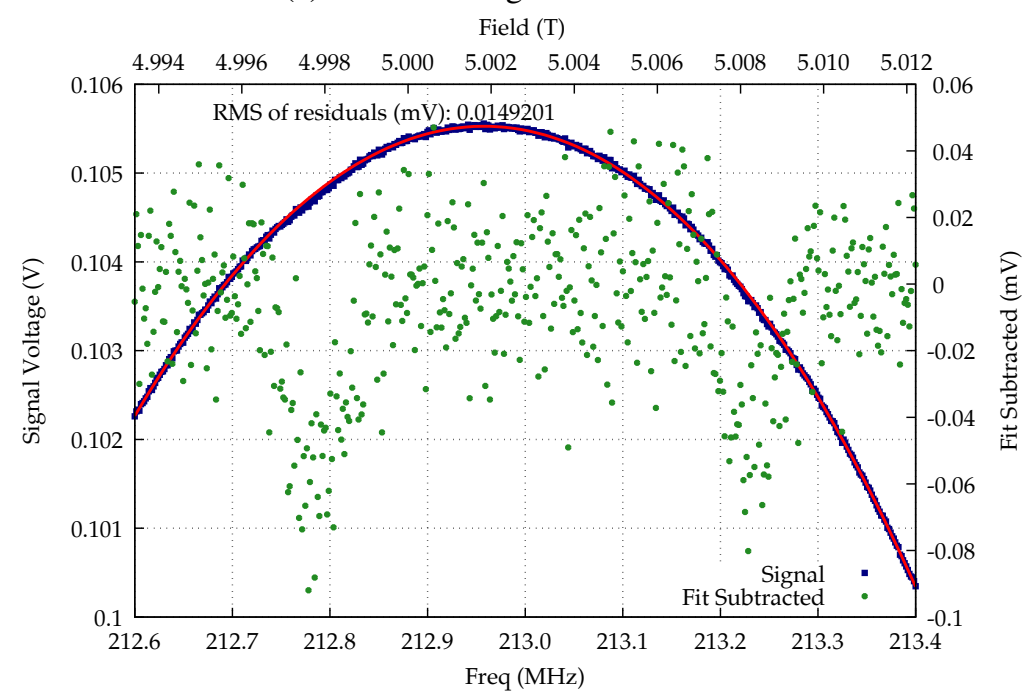

(b) Separated signals with the shims on.

Figure 5: Thermal equilibrium signals from two adjacent sample cells coupled to NMR coils in parallel. Blue points show the raw signal, the red curve is a fit to the Q-curve background, and green points show the signal with the baseline fit subtracted. The $\mathrm{x}$-axis is shown both as the NMR frequency and the magnetic field that corresponds to that Larmor frequency for protons.

\section{Conclusions and Outlook}

These measurements represent a continuing effort to improve the continuous NMR system used with polarized solid targets at Jefferson Lab. The demonstration of opposing polarized cells using a single microwave frequency is an important step, particularly considering the test was accomplished with epoxy at high temperatures. Further NMR development is centering on the renovation and replacement of the aging Liverpool Q-meter systems. With the collaboration of the JLab Fast Electronics group, we are testing a new, prototype Q-meter, following the original 


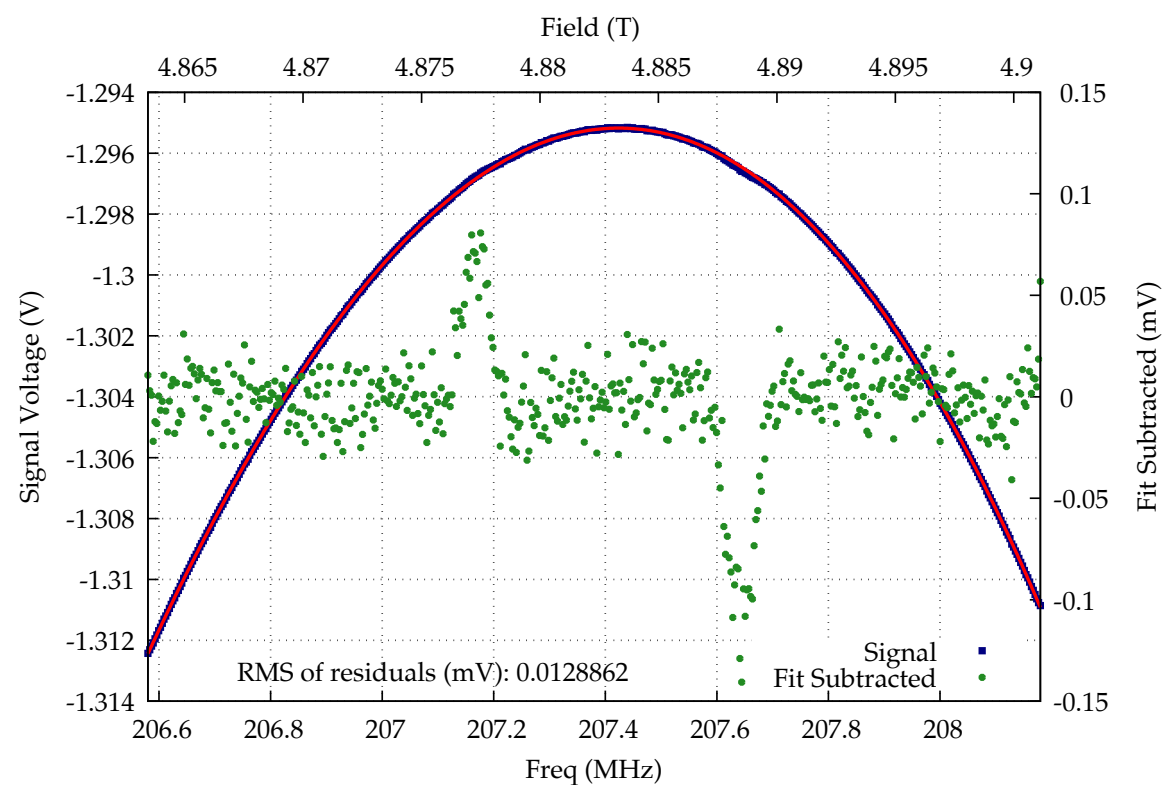

Figure 6: Polarization signal showing opposing polarizations in two cells held in the two shim regions, driven by a single microwave frequency.

closely but using modern components (much like the Bochum effort [13]). Once a dependable modern copy is produced, we will incorporate improvements such as internal phase shifting and quadrature measurement with a second mixer. We hope to further reduce noise by including the DAQ inside a new Q-meter chassis design.

\section{Acknowledgments}

Many thanks to collaborator V. Lagerquist of Old Dominion University for her indispensable contributions, including the winding and simulation of shim coils. This material is based on work supported by the U.S. Department of Energy, Office of Science, Office of Nuclear Physics under contract DE-AC05-06OR23177.

\section{References}

[1] D. G. Crabb and W. Meyer, Solid polarized targets for nuclear and particle physics experiments, Annual Review of Nuclear and Particle Science 47 (1997) 67.

[2] G. Court, D. Gifford, P. Harrison, W. Heyes and M. Houlden, A high precision q-meter for the measurement of proton polarization in polarised targets, Nuclear Instruments and Methods in Physics Research Section A 324 (1993) 433.

[3] C. Keith, A dynamically polarized target for CLAS12, in Proceedings of the XVII International Workshop on Polarized Sources, Targets \& Polarimetry. 16-20 October, 2017 Kaist, South Korea (PSTP2017)., 2017, https://pos.sissa.it/324/008/pdf.

[4] D. Adams et al., The polarized double cell target of the smc, Nuclear Instruments and Methods in Physics Research Section A 437 (1999) 23. 
[5] K. Kondo et al., Polarization measurement in the compass polarized target, Nuclear Instruments and Methods in Physics Research Section A 526 (2004) 70.

[6] P. McKee, Observations of radiation damage and recovery in ammonia targets, Nuclear Instruments and Methods in Physics Research Section A 526 (2004) 60 .

[7] J. Maxwell et al., Design and performance of the spin asymmetries of the nucleon experiment, Nuclear Instruments and Methods in Physics Research Section 885 (2018) 145.

[8] G. Court, M. Houlden, S. Bultmann, D. Crabb, D. Day, Y. Prok et al., High precision measurement of the polarization in solid state polarized targets using $\mathrm{nmr}$, Nuclear Instruments and Methods in Physics Research Section A 527 (2004) 253.

[9] E. J. Veenendaal, R. Hulstman and H. B. Brom, A frequency-modulated q-meter for very low-temperature nmr experiments, Journal of Physics E: Scientific Instruments 16 (1983) 649.

[10] MACOM, "Macom MA46 series GaAs tuning varactors."

[11] Y. Noda, T. Kumada, D. Yamaguchi and S. Shamoto, Thermosetting polymer for dynamic nuclear polarization: Solidification of an epoxy resin mixture including tempo, Nuclear Instruments and Methods in Physics Research Section A 776 (2015) 8.

[12] J. Maxwell, NMR Polarization Measurements for Jefferson Lab's Solid Polarized Targets, in Proceedings of the XVII International Workshop on Polarized Sources, Targets \& Polarimetry. 16-20 October, 2017 Kaist, South Korea (PSTP2017)., https://pos.sissa.it/324/011/pdf.

[13] J. Herick, Development of a new Q-meter module, in Proceedings, 16th International Workshop on Polarized Sources, Targets, and Polarimetry (PSTP 2015): Bochum, Germany, September 14-18, 2015, vol. PSTP2015, p. 011, 2016, https://pos.sissa.it/243/011/pdf. 